\title{
Malaria Modeling in the Era of Eradication
}

\author{
Thomas A. Smith, Nakul Chitnis, Melissa Penny, and Marcel Tanner \\ Department of Epidemiology and Public Health, Swiss Tropical and Public Health Institute, \\ Basel CH 4002, Switzerland; and University of Basel, Basel CH 4001, Switzerland \\ Correspondence: thomas-a.smith@unibas.ch
}

\begin{abstract}
Mathematical models provide the essential basis of rational research and development strategies in malaria, informing the choice of which technologies to target, which deployment strategies to consider, and which populations to focus on. The Internet and remote sensing technologies also enable assembly of ever more relevant field data. Together with supercomputing technology, this has made available timely descriptions of the geography of malaria transmission and disease across the world and made it possible for policy and planning to be informed by detailed simulations of the potential impact of intervention programs. These information technology advances do not replace the basic understanding of the dynamics of malaria transmission that should be embedded in the thinking of anyone planning malaria interventions. The appropriate use of modeling may determine whether we are living in an era of hubris or indeed in an age of eradication.
\end{abstract}

$\mathrm{T}^{\mathrm{h}}$ he quantitative understanding of malaria dynamics that is essential for rational planning of intervention programs needs to be supported by mathematical models. These models must address considerably more challenges and factors than is generally the case for directly transmitted pathogens or for noncommunicable diseases. The malaria life cycle is complicated, as are host immune responses to the parasite and the consequent pathogenesis. The diversity of Anopheles vectors, with their own complex life cycles and distinct ecologies also complicate malaria dynamics, resulting in an enormous variety of situations and of potential intervention strategies. Evaluating all of these empirically in field trials is simply impossible, especially because effects can occur over long timescales. Malaria is, thus, an archetypical example of a complex system, in which mathematical mod- eling is needed for studying the magnitude of effects of different components and for explaining their interactions and for making predictions about their behavior and how this depends on multiple drivers.

The need for mathematical models that simultaneously consider the effects of these different factors was recognized by Ross (1911), not long after he elucidated the transmission cycle, making malaria one of the first infectious diseases to be analyzed mathematically. Ross showed that attacking the Anopheles mosquito could be effective in controlling malaria but that it was not necessary to completely eliminate the vector to eliminate the infection: This motivated substantial efforts in larval control in the early 20th century. The development of indoor residual spraying (IRS) using DDT in the mid20th century as mainstream of the World Health

Editors: Dyann F. Wirth and Pedro L. Alonso

Additional Perspectives on Malaria: Biology in the Era of Eradication available at www.perspectivesinmedicine.org

Copyright (C) 2017 Cold Spring Harbor Laboratory Press; all rights reserved; doi: 10.1101/cshperspect.a025460

Cite this article as Cold Spring Harb Perspect Med 2017;7:a025460 
T.A. Smith et al.

Organization (WHO) Global Malaria Eradication Program (GMEP) stimulated further development of mathematical models of malaria interventions, mainly led by George Macdonald (1956). The central insight of these models is that, despite the complexity of malaria transmission, many of the factors affecting it can be quantified in a single equation for $R_{0}$, the basic reproductive number (Fig. 1). This measures how many infectious humans could be expected to arise from a single infectious human, assuming all other humans and mosquitoes are susceptible. Using this equation, it is straightforward to compare impact of intervention at different stages of the malaria cycle and show that, assuming all other things being equal, killing adult female mosquitoes has a much greater transmission effect than an equivalent attack on any other stage in the life cycle of the parasite or vector considered in the model (Fig. 1). IRS with DDT provided an effective way of achieving exactly that, and armies of malaria workers were trained to implement what was perceived of as a one-size-fits-all campaign with well-defined activities and timelines (Pampana 1963). Although these efforts were successful in many parts of the world, they did not lead to interruption of transmission in every setting. A lesson of this was that malaria control and elimination require additional interventions, especially in Africa, where transmission is most intense.

\section{BEYOND ROSS-MACDONALD}

Much malaria modeling has been built on the Ross-Macdonald model (Smith et al. 2014) but the key insight remains valid: The most traction will generally be achieved in reducing transmission by targeting the adult female vector. This is borne out by the tremendous success of scaleup of insecticide-treated nets (ITNs) in reducing transmission across Africa in the last decade (Bhatt et al. 2015). In general, interventions that target malaria in humans need to achieve very high efficacy, coverage, and long duration if they are to be comparable with vector control in their impacts on transmission. So far, we do not have any such interventions.
At the same time, the basic Ross-Macdonald framework does not consider all elements even of the life cycle of the Anopheles mosquito or how best to reduce adult mosquito survival. The $R_{0}$ concept, elegant in its simplicity, is more complicated when heterogeneities in space and time (e.g., seasonality) need to be considered (Diekmann et al. 1990; Heffernan et al. 2005), and the aspects of malaria dynamics considered by Macdonald are only a subset of those that need modeling. Although computer technology continually increases the computational possibilities, modeling malaria in a complex, dynamic, rapidly changing world finds ever more challenges. Some of them are discussed below.

\section{Surveillance Response}

The models available did not address all the challenges of malaria programs recognized in the mid-20th century. In general, the impact on transmission of a malaria intervention program is maximal shortly after scale-up (Smith and Schapira 2012), so IRS programs were originally intended to be time-limited to achieve very rapid interruption of transmission (Pampana 1963; Macdonald and Göckel 1964). Once malaria transmission is very low or has been interrupted, an effective surveillance system is essential, and if significant residual vectorial capacity remains then the surveillance must have a reactive component consisting of well-tailored (to a given epidemiological setting) and validated integrated response packages that is effective in preventing transmission from imported cases. This can be very expensive and difficult to operate in tropical areas. Just as with transmission reduction, models are needed to identify the critical elements of surveillance-response approaches. This remains an underdeveloped area of modeling.

\section{Temporal Dynamics}

The timelines for elimination programs established for in the mid-20th century (Macdonald and Göckel 1964) no longer seem realistic. This is partly a consequence of the fact that recent intervention programs generally do not achieve maximal coverage at the start, and transmission- 


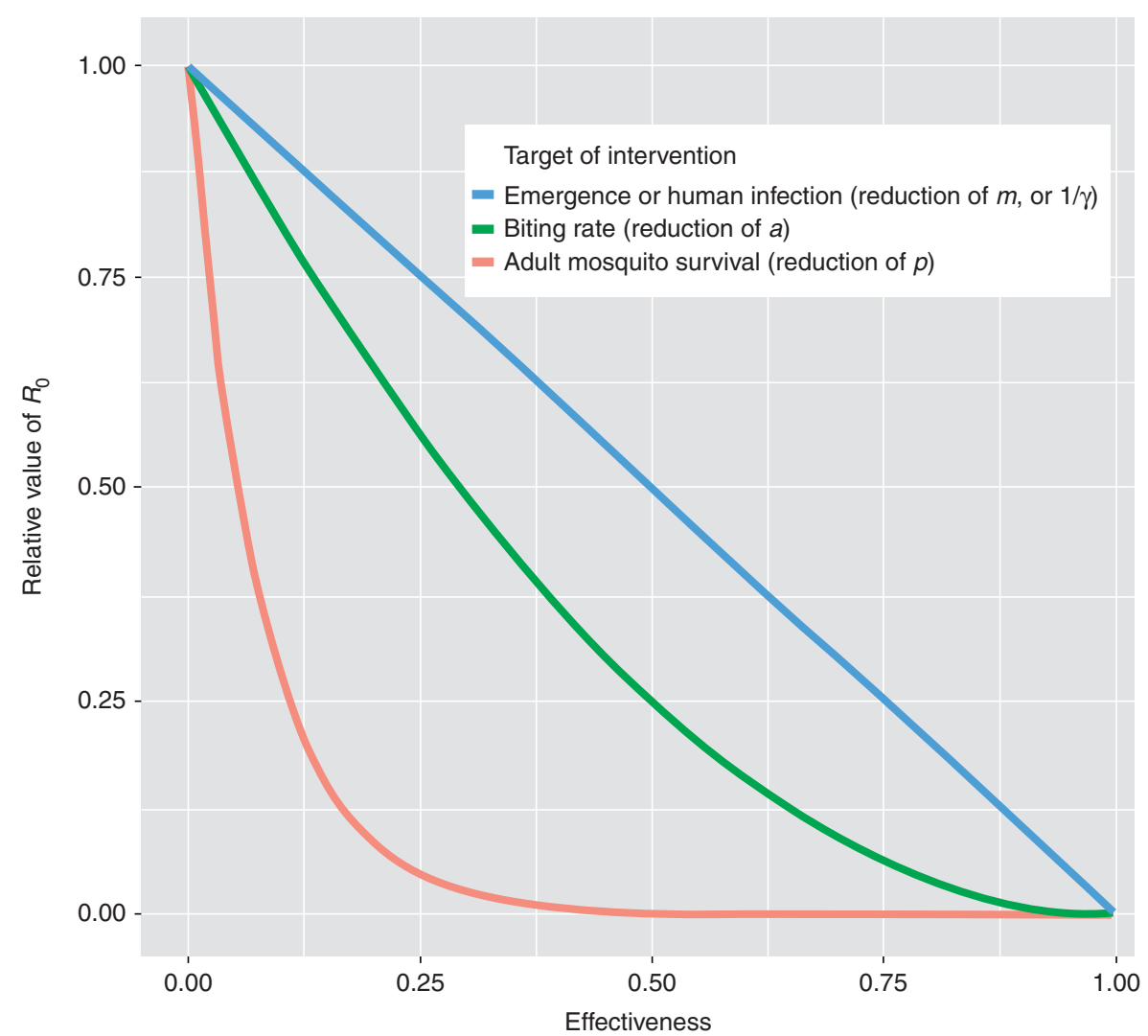

Figure 1. Sensitivity of $R_{0}$ to changes in mosquito density, biting rate, and mosquito survival calculated for the Ross-Macdonald model (based on Koella 1991). Macdonald's formula for $R_{0}$ is

$$
R_{0}=\frac{m a^{2} b c p^{\tau}}{-\gamma \log (p)},
$$

where the different parameters are $m=$ the number of female mosquitoes per human host, $a=$ the number of bites per mosquito per day, $b=$ the probability of transmitting infection from an infectious mosquito to a human (per bite), $c=$ the probability of transmission of infection from an infectious human to a mosquito (per bite), $\gamma=$ rate of recovery of humans from infectiousness (equivalently, $1 / \gamma$, duration of human infectiousness), $p=$ the daily survival of adult mosquitoes, and $\tau=$ the period required for development of sporozoites from infection of the mosquito. Changes in parameter values are represented as the efficacy of shown factors relating to the original setting (e.g., an efficacy of $50 \%$ corresponds to a multiplication of $m$, or $1 / \gamma$ by $0.5 ; m$ or $1 / \gamma$ enter the equation for the basic reproductive number linearly); therefore, this efficacy corresponds to a $50 \%$ decrease in reproductive number. Biting rate, $a$, enters the reproductive number quadratically, so that an efficacy of $50 \%$ in reducing this leads to reduction in $R_{0}$ to $0.5^{2}=0.25$ times its original value. Efficacy in adulticiding of $50 \%$ corresponds to a $50 \%$ reduction in survival per unit time. This enters the formula for $R_{0}$ as a power function so decreases in this lead to the largest changes in $R_{0}$.

reducing interventions are consequently applied for long periods. The reasons are many: $R_{0}$ for Plasmodium falciparum can be extremely high (Smith et al. 2007), characteristics of the mosquitoes (such as exophily) limiting the effective- ness of vector control measures; the populations needing coverage may be large, and therefore likely to encompass heterogeneities in transmission and in the health system leading to potentially reduced impact of interventions; and 
T.A. Smith et al.

more gradual scale-up of impact is likely to be achieved with ITNs as the main transmissionreducing intervention rather than IRS. As malaria transmission is brought down, it is essential for the control and elimination program to reorient mainly toward intensified surveillance-response approaches as immunity in the population is gradually reduced.

Understanding of the temporal dynamics induced by long-term programs and optimizing decision rules for program adjustments require consideration of the time dimension of the different interventions. This depends on the technical characteristics of the intervention, on the induced effects on natural immunity, and on external drivers of seasonal and interannual variations in malaria transmission such as rainfall and temperature. Population models of malaria incorporating natural immunity are thus needed to predict these dynamics. There is a substantial mathematical literature on malaria immunity based around susceptible-exposedinfectious-recovered dynamics (Ngwa and Shu 2000; Mandal et al. 2011; Reiner et al. 2013), but these fail to capture fundamental aspects of what is known about superinfection in both P. falciparum and Plasmodium vivax. Other relatively simple compartment models (Dietz et al. 1974; Anderson and May 1991; Gupta and Day 1994) also failed to capture key aspects of malaria immunity, leading more recently to the use of a new generation of relatively complex compartment models and microsimulations (see below) (Smith et al. 2006, 2012; Griffin et al. 2010; Eckhoff 2011).

\section{Spatial Variation and Movement}

Because underlying transmission potential is everywhere highly heterogeneous and programs rarely achieve synchronous scale-up of vector control, transmission persists in pockets or foci. This reduces the impact of untargeted interventions but makes them more effective if they can target highly exposed hosts (Woolhouse et al. 1997). There is a substantial literature on extensions of models of vector-borne disease to take into account transmission heterogeneity following on from key publications three decades ago (Dye and Hasibeder 1986). Heterogeneity in transmission potential is, however, not the only kind of heterogeneity needing to be considered in models of public health outcomes of malaria interventions (Ross and Smith 2010). It is particularly important to consider all such heterogeneities in predicting the outcome of programs that aim to eliminate the infection. Models of average outcomes (such as, e.g., Tatem et al. 2010) generally provide optimistic predictions because it is the challenging foci, not the average, that determine whether the infection is eliminated.

Other models consider the impact of movements of both humans and vector populations. These models have ranged from general theoretical models that have been used to show that human movement can lead to persistence of malaria where it would not have otherwise persisted (Cosner et al. 2009) to more detailed models that can provide estimates of numbers of imported cases in particular locations (Tatem et al. 2009; Le Menach et al. 2011) or help to plan spatial targeting of vector control interventions (Lutambi et al. 2013).

\section{Stochasticity}

Until the last decade, most widely used mathematical models for malaria transmission, including Macdonald's formulation and later models incorporating immunity (Dietz et al. 1974), have been deterministic continuous state models that consider average status in an undefined size of population. Such models cannot capture chance or stochastic phenomena that are particularly important when transmission rates are low. In particular, a deterministic system can achieve a stable state with very low transmission, although a stochastic simulation (or the real world) will sooner or later achieve extinction if average transmission is very low, irrespective of the estimated basic reproduction number.

Population size is critical for stochastic loss but has so far not been adequately addressed in malaria modeling. Even stochastic implementations of models that allow unlimited superinfection, such as those of Smith et al. (2006) and 
Walker et al. (2015), cannot capture what happens in small foci. When parasite populations are small, a large proportion of inoculations represent recurrent exposure to the same parasite genotypes, and malaria therapy data show that this is less likely to result in infection than is inoculation with heterologous parasites (Molineaux et al. 2002; Collins et al. 2004). Because reduction of transmission leads to progressive fragmentation of parasite (or vector) populations into discrete foci, this in turn will lead to collapse of local genetic diversity. This is likely to be a key stage in interrupting transmission, but modeling of this is still poorly developed. We have, for instance, no good estimates of the minimum population size for endemic malaria (either P. falciparum or P. vivax).

\section{Within-Host Dynamics}

Just as there are variations in the vulnerability of the parasite at different stages in the transmission cycle between the mosquito vector and the human, there are also variations between stages within the human and within the mosquito in suitability as intervention targets. One driver of this is the enormous variation in biomass between different parasite stages (Sinden et al. 2007), which provides an indication of which stages might be most vulnerable within the host, because they represent a bottleneck in the parasite life cycle. This is potentially key information for choice of drug and vaccine targets. To understand how this translates into potential impacts of interventions needs models of the various density-dependent regulation processes, including the effects of the immune system (e.g., the within-host model of Molineaux et al. 2001).

\section{New Interventions}

The new era initiated by the 2007 Gates Malaria Forum (Roberts and Enserink 2007) is not just a time of appeals for disease eradication, it is also a time of technological change at an unprecedented rate. There is consensus that eradication is impossible without a new generation of tools and strategies (Alonso et al. 2011), therefore, at present, the only rational global eradication strategy is to focus on identifying and developing the transformative technologies that are needed. Some of these technologies will be improved versions of existing interventions, such as ITNs, drugs, and insecticides. Others will be novel deployment strategies, tools for more efficient dissemination of information (such as mHealth tools), and ways of integrating these such as new strategies for surveillance response.

Modeling should be an essential component of establishing target product profiles (TPPs) for vaccine, drugs, and diagnostics in the research and development process aiming at malaria elimination and eradication. Before product developers lock themselves into inappropriate development pathways, models need to be applied to address the questions of which deployment strategies to consider and how interventions, including both new and established ones, might best be combined with each other in integrated packages. Models should be used to evaluate the potential of deployment strategies before deciding whether to embark on fullscale field trials. For instance, modeling of vaccine combinations suggests that duration of protection, the choice of target group, the timing, and the deployment strategy are as important as antigen selection. Multiple components targeting the same parasite stage are likely to have more impact than multistage vaccines if they can achieve higher efficacy and longer duration of protection, and this needs to be considered in trial design. Transmission reduction could theoretically be achieved with vaccines targeting any parasite stage, but to substantially reduce transmission or achieve elimination they will need to reach very large proportions of the population (Penny et al. 2008). Vaccines with profiles similar to the recently evaluated RTS,S might be an important component of intervention packages designed for elimination in lowtransmission settings if deployed across a wide age range.

\section{Mosquito Ecology}

Because of the importance of killing adult mosquitoes indicated by Macdonald's analysis, novel vector control interventions represent a par- 
T.A. Smith et al.

ticularly promising avenue, but there is an implicit assumption in Macdonald's analysis that the emergence of the next generation of mosquitoes will not be affected by adulticiding interventions. Different models are thus needed in planning interventions that hope to eliminate the mosquitoes. Such technologies are urgently needed because elimination of malaria from much of the world will require technologies that target outdoor biting and resting mosquitoes that are refractory to ITNs and IRS. These include models of larval competition (White et al. 2011; Smith et al. 2013) and of the systems needed to drive through the population interventions based on genetically modified mosquitoes (Boete and Koella 2002) or entomopathogens (Hancock et al. 2008) and of various kinds of insecticidal baits (Okumu et al. 2010).

\section{Resistance and Evolutionary Models}

A basic principle in evolutionary ecology is that interventions against an organism will increase the frequency of genetic variants that evade them. This applies to all malaria interventions, most obviously (but not exclusively) leading to drug (Escalante et al. 2009) and insecticide resistance. The best strategies for managing this are not always obvious (Hastings 2010). Evolutionary changes to evade interventions are not always harmful, and evolutionary models can help identify interventions that may push the evolution of vectors or parasites in directions that make them less harmful. For instance, by increasing the relative fitness of high fecundity, short lifespan mosquitoes (Ferguson et al. 2012) and increasing the relative fitness of zoophilic vectors, ITNs may select not only insecticide-resistant mosquitoes but also ones that are less competent malaria vectors. However, some studies have also shown that resistance to insecticide in mosquitoes could increase their susceptibility to Plasmodium infection (Ndiath et al. 2014) and further increase malaria transmission. Well-calibrated population genetics models of the evolution of resistance of both parasites (to drugs) and mosquitoes (to insecticides) are needed that can capture both the drivers of the evolution of resistance and its impact on malaria transmission.

\section{Prediction and Microsimulation Models}

At the same time as modeling new interventions, there is a need to reduce disease burden as efficiently as possible using existing tools. This requires guidance on the likely impact in particular places of integrated programs consisting of an array of specific intervention deployments. Global resources allocated for malaria programs remain far below what is required to achieve universal coverage of all recommended interventions (WHO 2015b), making it essential to carry out economic evaluations for optimizing budget allocations, as well as epidemiological ones. Economic evaluations of health interventions have generally used rather simple static models of disease dynamics, but the recognition that the dynamic processes of feedback effects can radically modify cost-effectiveness has motivated linking of costing data with quantitative results from dynamic models of epidemiology. Economic evaluation of improvements in case management and in health systems in general remains a challenge.

At the policy level, modeling is again seen as a necessary step in the rollout of a new intervention strategy. This has motivated the development of complex simulation models that aim to capture as many factors as possible that might influence quantitative outcomes of intervention programs (Smith et al. 2006, 2012; Griffin et al. 2010; Eckhoff 2011). The recent publication of results from the Phase III trial of the RTS,S vaccine (RTS,S Clinical Trials Partnership 2015) has been accompanied by a major exercise to apply a multimodel ensemble to predict the impact of specific intervention strategies (Penny et al. 2015b). This provided an unprecedented opportunity to evaluate the degree of consensus among simulation models. Despite differences in assumptions and model structures the models included are in broad agreement on the strategies and settings in which RTS,S would have the greatest public health impact and cost-effectiveness and hence on recommendations on use of the vaccine. 
Quantitative predictions of impact and cost-effectiveness make it possible to compare both between malaria interventions and with comparable interventions against other diseases. For instance, RTS,S is predicted to be relatively cost-effective compared with vaccines against other diseases, but may not be the most cost-effective way to reduce malaria burden. However, different interventions may be synergistic: RTS,S may be best used to reduce childhood malaria burden when implemented with existing high coverage of ITNs.

\section{GEOGRAPHICAL SPECIFICITY}

High-resolution open-access geographical databases of relevant data on human population distribution (Tatem et al. 2012), human and pathogen movement (Tatem 2014), and remote-sensed risk factors are becoming much more available (Bhatt et al. 2015). The malaria community now has particularly rich databases of geolocated parasitological survey data (Gething et al. 2011), which make it possible to use largely empirical sources to estimate disease burden. Mathematical models are still needed for assessing the feasibility of an elimination in a given setting as well as for projections of how interventions and specific intervention mixes will impact public health in the future. Finally, appropriate combination of mathematical and statistical approaches are providing a continually improving understand of how patterns of malaria and of interventions evolved in the recent past (Bhatt et al. 2015).

The availability of massive amounts of geolocation data means that modeling of the likely impacts of specific intervention programs (like RTS,S vaccination) in specific places and times has become feasible (Penny et al. 2015a), and these profuse data are available for calibration and validation of models. This sets a high bar for malaria modeling: Fitting complex models to multiple types of data is challenging, and model predictions are always likely to be unreliable at very high spatial resolution. The twin objectives of understanding the dynamics and making quantitative predictions can also be in conflict, because the push to include all relevant factors in a locally calibrated predictive model rapidly leads to complex behavior that can no longer be explained.

\section{FUTURE PERSPECTIVES}

The volume of data from remote sensing, available for parameterizing models of malaria in specific areas, will no doubt continue to grow massively. Electronic communications and data capture mean that disease surveillance systems will soon be accessing close to real-time data in cyberspace, enabling such models to propose optimized intervention responses tailored to particular places, at the appropriate level of spatial and temporal disaggregation.

If malaria is to be eradicated, modeling will need to contribute much more than datamining applications. Computers will not circumvent the need for humans to apply an understanding of the dynamics of malaria in considering which novel interventions to develop, in product development, and in designing and costing rational surveillance and intervention plans within national and regional strategies.

\section{CONCLUDING REMARKS}

Models are invaluable to support understanding of the impacts of interventions on malaria dynamics and represent a cornerstone for the implementation of the global technical strategy (WHO 2015a) at the global and national level. Some of these models can be built on the RossMacdonald framework. Just as an understanding of aerodynamics was needed by the astronauts who flew to the moon, the planners of malaria eradication need to understand these basic dynamics. However, rocket science based on physical laws is simple compared with disease eradication in complex biological systems. Multifarious phenomena need to be considered in malaria models.

All too often, models are seen as black boxes, and a considerable effort is required to understand them. Mathematical modeling is barely regulated, unlike most of the activities in pharmaceutical development and clinical trials, and the onus is on the modelers to communicate the 
T.A. Smith et al.

basic structure of their models and how they are fitted to data and validated and at the same time be clear about where there is consensus and where there are major uncertainties in their predictions. Sensitivity analyses and analysis of uncertainty and understanding its implications are essential components of any responsible prediction exercise. Modeling of the RTS,S vaccine represents one example in which there has been considerable attention to critical evaluation and validation of models, including documentation, quality control, and comprehensive review. Evaluation of the structural uncertainties in model predictions via multimodel ensembles (Smith et al. 2012; Cameron et al. 2015) or assessment of the consequences of uncertainties in parameter values are generally much more time-consuming and challenging than the modeling itself. It is especially difficult to convey this message to decision-makers who struggle to know what to do about uncertainty. So, to paraphrase the much-abused words of George Box, it is challenging for a nonspecialist to distinguish modeling that is useful from poor-quality modeling that may support misguided policies.

The science of complex systems, not least, systems biology, warns us that there are limits to the predictability of the events we are interested in. Elimination and eradication are extreme events, and extreme events are the most difficult to reliably predict. All those planning intervention strategies and making policy need not only heed projections of mostly likely or preferred scenarios but also understand that they are embarking on a road that is not, and cannot be, mapped out with a great deal of confidence and to be prepared if the best predictions of the route prove either greatly optimistic or unduly pessimistic.

Not all the unknowns make eradication harder. We also live in an era of mass extinction: another process full of uncertainties. Eradication is deliberate extinction, so models of ecological catastrophe hold lessons for disease eradication. The system of $P$. falciparum malaria transmitted by Afrotropical vectors is exquisitely adapted to the ecology of African subsistence agriculture, with people living in mud and thatch houses in intimate contact with an envi- ronment rich in Anopheles breeding sites. $P$. vivax also has a specific ecology, which facilitated its disappearance from the industrialized world. As the lifestyles supporting it disappear, both major species of human malaria may retreat from the rest of the world. Intelligent deployment of interventions could end the disease sooner.

\section{ACKNOWLEDGMENTS}

The authors have received support from the Bill and Melinda Gates Foundation, Grant OPP1032350, and acknowledge the contributions of their colleagues Katya Galactionova, Amanda Ross, Peter Pemberton-Ross, Diggory Hardy, Tobias Thüring, Flavia Camponovo, and Olivier Briet to the Swiss Tropical and Public Health (TPH) malaria-modeling program.

\section{REFERENCES}

Alonso PL, Brown G, Arevalo-Herrera M, Binka F, Chitnis C, Collins F, Doumbo OK, Greenwood B, Hall BF, Levine $\mathrm{MM}$, et al. 2011. A research agenda to underpin malaria eradication. PLoS Med 8: e1000406.

Anderson RM, May RM. 1991. Infectious diseases of humans: Dynamics and control. Oxford University Press, Oxford.

Bhatt S, Weiss DJ, Cameron E, Bisanzio D, Mappin B, Dalrymple U, Battle KE, Moyes CL, Henry A, Eckhoff PA, et al. 2015. The effect of malaria control on Plasmodium falciparum in Africa between 2000 and 2015. Nature 526: 207-211.

Boete C, Koella JC. 2002. A theoretical approach to predicting the success of genetic manipulation of malaria mosquitoes in malaria control. Malaria J 1: 3 .

Cameron E, Battle KE, Bhatt S, Weiss DJ, Bisanzio D, Mappin B, Dalrymple U, Hay SI, Smith DL, Griffin JT, et al. 2015. Defining the relationship between infection prevalence and clinical incidence of Plasmodium falciparum malaria: An ensemble model. Nat Commun 6: 8170.

Collins WE, Jeffery GM, Roberts JM. 2004. A retrospective examination of reinfection of humans with Plasmodium vivax. Am J Trop Med Hyg 70: 642-644.

Cosner C, Beier JC, Cantrell RS, Impoinvil D, Kapitanski L, Potts MD, Troyo A, Ruan S. 2009. The effects of human movement on the persistence of vector-borne diseases. $J$ Theor Biol 258: 550-560.

Diekmann O, Heesterbeek JA, Metz JA. 1990. On the definition and the computation of the basic reproduction ratio $R_{0}$ in models for infectious diseases in heterogeneous populations. J Math Biol 28: 365-382.

Dietz K, Molineaux L, Thomas A. 1974. A malaria model tested in the African savannah. Bull World Health Org 50: 347-357. 
Dye C, Hasibeder G. 1986. Population dynamics of mosquito-borne disease: Effects of flies which bite some people more frequently than others. Trans $R$ Soc Trop Med Hyg 80: 69-77.

Eckhoff PA. 2011. A malaria transmission-directed model of mosquito life cycle and ecology. Malaria J 10: 303.

Escalante AA, Smith DL, Kim Y. 2009. The dynamics of mutations associated with anti-malarial drug resistance in Plasmodium falciparum. Trends Parasitol 25: 557-563.

Ferguson HM, Maire N, Takken W, Lyimo IN, Briet O, Lindsay SW, Smith TA. 2012. Selection of mosquito life-histories: A hidden weapon against malaria? Malaria J 11: 106.

Gething PW, Patil AP, Smith DL, Guerra CA, Elyazar IR, Johnston GL, Tatem AJ, Hay SI. 2011. A new world malaria map: Plasmodium falciparum endemicity in 2010. Malaria J 10: 378.

Griffin JT, Hollingsworth TD, Okell LC, Churcher TS, White M, Hinsley W, Bousema T, Drakeley CJ, Ferguson NM, Basanez MG, et al. 2010. Reducing Plasmodium falciparum malaria transmission in Africa: A model-based evaluation of intervention strategies. PLoS Med 7: e1000324.

Gupta S, Day KP. 1994. A theoretical framework for the immunoepidemiology of Plasmodium falciparum malaria. Parasite Immunol 16: 361-370.

Hancock PA, Thomas MB, Godfray HC. 2008. An age-structured model to evaluate the potential of novel malariacontrol interventions: A case study of fungal biopesticide sprays. Proc Biol Sci 276: 71-80.

Hastings I. 2010. How artemisinin-containing combination therapies slow the spread of antimalarial drug resistance. Trends Parasitol 27: 67-72.

Heffernan JM, Smith RJ, Wahl LM. 2005. Perspectives on the basic reproductive ratio. J $R$ Soc Interface 2: 281-293.

Koella JC. 1991. On the use of mathematical models of malaria transmission. Acta Trop 49: 1-25.

Le Menach A, Tatem AJ, Cohen JM, Hay SI, Randell H, Patil AP, Smith DL. 2011. Travel risk, malaria importation and malaria transmission in Zanzibar. Sci Rep 1: 93.

Lutambi AM, Penny MA, Smith T, Chitnis N. 2013. Mathematical modelling of mosquito dispersal in a heterogeneous environment. Math Biosci 241: 198-216.

Macdonald G. 1956. Theory of the eradication of malaria Bull World Health Organ 15: 369-387.

Macdonald G, Göckel GW. 1964. The malaria parasite rate and interruption of transmission. Bull World Health Organ 31: 365-377.

Mandal S, Sarkar RR, Sinha S. 2011. Mathematical models of malaria-A review. Malaria J 10: 202.

Molineaux L, Diebner HH, Eichner M, Collins WE, Jeffery GM, Dietz K. 2001. Plasmodium falciparum parasitaemia described by a new mathematical model. Parasitology 122: $379-391$.

Molineaux L, Trauble M, Collins WE, Jeffery GM, Dietz K. 2002. Malaria therapy reinoculation data suggest individual variation of an innate immune response and independent acquisition of antiparasitic and antitoxic immunities. Trans R Soc Trop Med Hyg 96: 205-209.

Ndiath MO, Cailleau A, Diedhiou SM, Gaye A, Boudin C, Richard V, Trape JF. 2014. Effects of the $k d r$ resistance mutation on the susceptibility of wild Anopheles gambiae
Malaria Modeling in the Era of Eradication

populations to Plasmodium falciparum: A hindrance for vector control. Malaria J 13: 340.

Ngwa GA, Shu WS. 2000. A mathematical model for endemic malaria with variable human and mosquito populations. Math Comput Model 32: 747-763.

Okumu FO, Govella NJ, Moore SJ, Chitnis N, Killeen GF. 2010. Potential benefits, limitations and target productprofiles of odor-baited mosquito traps for malaria control in Africa. PLoS ONE 5: e11573.

Pampana E. 1963. A textbook of malaria eradication. Oxford University Press, London.

Penny MA, Maire N, Studer A, Schapira A, Smith TA. 2008. What should vaccine developers ask? Simulation of the effectiveness of malaria vaccines. PLoS ONE 3: e3193.

Penny MA, Galactionova E, Tarantino M, Tanner M, Smith T. 2015a. The public health impact of malaria vaccine RTS,S in malaria endemic Africa: Country-specific predictions using 18 month follow-up Phase III data and simulation models. BMC Med 13: 170.

Penny MA, Verity R, Bever C, Sauboin C, Galactionova E, Flasche S, White MT, Wenger EA, van der Velde N, Pemberton-Ross $\mathrm{P}$, et al. 2015b. The public health impact and cost-effectiveness of malaria vaccine RTS,S/AS01: A systematic comparison of predictions and four mathematical models. Lancet 23: 367-375.

Reiner RC Jr, Perkins TA, Barker CM, Niu T, Chaves LF, Ellis AM, George DB, Le MA, Pulliam JR, Bisanzio D, et al. 2013. A systematic review of mathematical models of mosquito-borne pathogen transmission: 1970-2010. J R Soc Interface 10: 20120921.

Roberts L, Enserink M. 2007. Malaria. Did they really say ... eradication? Science 318: 1544-1545.

Ross R. 1911. The prevention of malaria, 2nd ed. John Murray, London.

Ross A, Smith T. 2010. Interpreting malaria age-prevalence and incidence curves: A simulation study of the effects of different types of heterogeneity. Malaria J 9: 132.

RTS,S Clinical Trials Partnership. 2015. Efficacy and safety of RTS,S/AS01 malaria vaccine with or without a booster dose in infants and children in Africa: Final results of a phase 3 , individually randomised, controlled trial. Lancet 386: $31-45$.

Sinden RE, Dawes EJ, Alavi Y, Waldock J, Finney O, Mendoza J, Butcher GA, Andrews L, Hill AV, Gilbert SC, et al. 2007. Progression of Plasmodium berghei through Anopheles stephensi is density-dependent. PLoS Pathog 3: e195.

Smith T, Schapira A. 2012. Reproduction numbers in malaria and their implications. Trends Parasitol 28: 3-8.

Smith T, Killeen GF, Maire N, Ross A, Molineaux L, Tediosi F, Hutton G, Utzinger J, Dietz K, Tanner M., 2006. Mathematical modeling of the impact of malaria vaccines on the clinical epidemiology and natural history of Plasmodium falciparum malaria: Overview. Am J Trop Med Hyg 75: $1-10$.

Smith DL, McKenzie FE, Snow RW, Hay SI. 2007. Revisiting the basic reproductive number for malaria and its implications for malaria control. PLoS Biol 5: e42.

Smith T, Ross A, Maire N, Chitnis N, Studer A, Hardy D, Brooks A, Penny M, Tanner M. 2012. Ensemble modeling of the likely public health impact of a pre-erythrocytic malaria vaccine. PLoS Med 9: e1001157. 
T.A. Smith et al.

Smith DL, Perkins TA, Tusting LS, Scott TW, Lindsay SW 2013. Mosquito population regulation and larval source management in heterogeneous environments. PLoS ONE 8: e71247.

Smith DL, Perkins TA, Reiner RC Jr, Barker CM, Niu T, Chaves LF, Ellis AM, George DB, Le MA, Pulliam JR, et al. 2014. Recasting the theory of mosquito-borne pathogen transmission dynamics and control. Trans $R$ Soc Trop Med Hyg 108: 185-197.

Tatem AJ. 2014. Mapping population and pathogen movements. Int Health 6: 5-11.

Tatem AJ, Qiu Y, Smith DL, Sabot O, Ali AS, Moonen B. 2009. The use of mobile phone data for the estimation of the travel patterns and imported Plasmodium falciparum rates among Zanzibar residents. Malaria J 8: 287.

Tatem AJ, Smith DL, Gething PW, Kabaria CW, Snow RW, Hay SI. 2010. Ranking of elimination feasibility between malaria-endemic countries. Lancet 376: 1579-1591.

Tatem AJ, Adamo S, Bharti N, Burgert CR, Castro M, Dorelien A, Fink G, Linard C, John M, Montana L, et al. 2012. Mapping populations at risk: Improving spatial demo- graphic data for infectious disease modeling and metric derivation. Popul Health Metr 10: 8.

Walker PG, White MT, Griffin JT, Reynolds A, Ferguson NM, Ghani AC. 2015. Malaria morbidity and mortality in Ebola-affected countries caused by decreased healthcare capacity, and the potential effect of mitigation strategies: A modelling analysis. Lancet Infect Dis 15: 825 832.

White MT, Griffin JT, Churcher TS, Ferguson NM, Basanez MG, Ghani AC. 2011. Modelling the impact of vector control interventions on Anopheles gambiae population dynamics. Parasit Vectors 4: 153.

Woolhouse ME, Dye C, Etard JF, Smith T, Charlwood JD, Garnett GP, Hagan P, Hii J, Ndhlovu PD, Quinnell RJ, et al. 1997. Heterogeneities in the transmission of infectious agents: Implications for the design of control programs. Proc Natl Acad Sci 94: 338-342.

WHO. 2015a. Global technical strategy for malaria 2016 2030. World Health Organization, Geneva.

WHO. 2015b. World malaria report 2014. World Health Organization, Geneva. 


\section{$\&_{\mathrm{CSH}}^{\infty} \&$ Cold Spring Harbor

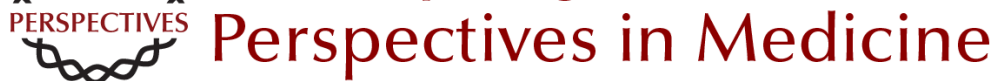

\section{Malaria Modeling in the Era of Eradication}

Thomas A. Smith, Nakul Chitnis, Melissa Penny and Marcel Tanner

Cold Spring Harb Perspect Med 2017; doi: 10.1101/cshperspect.a025460 originally published online December 21, 2016

\section{Subject Collection Malaria: Biology in the Era of Eradication}

Modern Vector Control

Neil F. Lobo, Nicole L. Achee, John Greico, et al.

Anopheline Reproductive Biology: Impacts on Vectorial Capacity and Potential Avenues for

Malaria Control Sara N. Mitchell and Flaminia Catteruccia

Current and Future Prospects for Preventing Malaria Transmission via the Use of Insecticides Hilary Ranson

Molecular Signaling Involved in Entry and Exit of Malaria Parasites from Host Erythrocytes Shailja Singh and Chetan E. Chitnis

Vaccines to Accelerate Malaria Elimination and Eventual Eradication Julie Healer, Alan F. Cowman, David C. Kaslow, et al.

Immune Responses in Malaria Carole A. Long and Fidel Zavala

Antimalarial Drug Resistance: A Threat to Malaria Elimination Didier Menard and Arjen Dondorp

Malaria during Pregnancy Michal Fried and Patrick E. Duffy
Malaria Pathogenesis Danny A. Milner, Jr.

Determinants of Malaria Transmission at the Population Level Teun Bousema and Chris Drakeley

\section{Host Cell Tropism and Adaptation of Blood-Stage Malaria Parasites: Challenges for Malaria}

Elimination Caeul Lim, Selasi Dankwa, Aditya S. Paul, et al.

Malaria Transmission and Prospects for Malaria Eradication: The Role of the Environment Marcia C. Castro

The Biology of Plasmodium vivax John H. Adams and Ivo Mueller

Malaria Genomics in the Era of Eradication Daniel E. Neafsey and Sarah K. Volkman

Malaria Epigenetics Alfred Cortés and Kirk W. Deitsch

Malaria Parasite Liver Infection and Exoerythrocytic Biology Ashley M. Vaughan and Stefan H.I. Kappe

For additional articles in this collection, see http://perspectivesinmedicine.cshlp.org/cgi/collection/ 